\title{
Role of Cardiac MRI in Assessment Non-Ischemic Cardiomyopathy
}

\author{
Mostafa Fadel Sonbol, Mahmoud Ibrahim Elshamy ", Anas Mohamed Sabry Elhadary \\ Department of Radiology, Faculty of Medicine, Al-Azhar University, Cairo, Egypt \\ Email address: \\ Prof.ms@hotmail.com (M. F. Sonbol), Elshamy.radio@gmail.com (M. I. Elshamy),Anaselhadary55@gmail.com (A. M. S. Elhadary) \\ ${ }^{*}$ Corresponding author
}

\section{To cite this article:}

Mostafa Fadel Sonbol, Mahmoud Ibrahim Elshamy, Anas Mohamed Sabry Elhadary. Role of Cardiac MRI in Assessment Non-Ischemic Cardiomyopathy. International Journal of Medical Imaging. Vol. 9, No. 1, 2021, pp. 72-78. doi: 10.11648/j.ijmi.20210901.17

Received: February 5, 2021; Accepted: February 23, 2021; Published: March 3, 2021

\begin{abstract}
Background: cardiomyopathies include a broad spectrum of myocardial problems which can affect the heart either a primary disease process or as part of a systemic disorder, which may be complicated by heart failure or death. Objective: the aim of the study to identify the role of cardiac magnetic resonance imaging to diagnose the different various types of cardiomyopathy. Patients and Methods: this study included 50 patients with suspected different types of non-ischemic cardiomyopathies referred from outpatient clinic of Cardiology at Al-Hussein University hospital from November 2018 to July 2019. Results: all patients showed mild to moderate mitral regurge (100\% of cases), tricuspid regurge was seen in 3 cases ( $75 \%$ of cases) and aortic regurge was seen in 4 cases (50\% of cases). The most value of MRI study of these patients was to differentiate whether if heart failure is related to 1ry dilated cardiomyopathy or other causes such as ischemia as they have different management in each group. Conclusion: CMR is a valuable (minimally or non-invasive) imaging technique in patients with suspected or known to have non-ischemic cardiomyopathies. It helps to identify the underlying cause, prognosis and responsiveness to therapy and affect management as well follow-up and most recently as a preventive diagnostic tool.
\end{abstract}

Keywords: MRI, CMR, Ischemic Cardiomyopathy

\section{Introduction}

Cardiomyopathy is characterized as a myocardial issue in which the heart muscle is primarily and practically strange without coronary illness, hypertension, valvular coronary illness and intrinsic coronary illness adequate to cause the noticed myocardial anomaly [1].

In the investigation work up for a presumed cardiomyopathy, ordinarily a significant number of tests are performed, going from the more daily practice and direct tests including ECG, echocardiogram, and exercise treadmill, to the more invasive including coronary angiography, electrophysiological considers, and endomyocardial biopsy. It is critical to distinguish a potentially treatable substrate and afterward to separate patients for treatment including thought of an implantable defibrillator (ICD) or heart resynchronization device [2].

In this survey, Non-Ischaemic Cardiomyopathies (NICMP) are drawn closer from a clinical perspective laying out the indicative and prognostic job of CMR as a basic piece of the clinical algorithm [2].

Cardiovascular magnetic resonance (CMR) is an important apparatus for assessment of patients with, or in danger for, cardiovascular breakdown, clinical management, and decision. Through its ability to characterize the myocardium by using multiple different imaging parameters, it provides insight into the etiology of the underlying heart failure and its prognosis [2].

CMR is widely accepted as the reference standard for quantifying chamber size and ejection fraction. Also, tissue characterization techniques such as late gadolinium enhancement (LGE) and other parameters such as T1 mapping, both native and with measurement of extracellular volume fraction; T2 mapping; and T2* mapping have been validated against histological findings in a wide range of clinical scenarios. Specifically, the example of LGE in the myocardium can help decide the basic etiology of the cardiovascular breakdown. The presence and degree of LGE decide guess in a large number of the non-ischemic cardiomyopathies. The utilization of CMR should increment 
as its utility in portrayal and evaluation of anticipation in cardiomyopathies is progressively perceived [3].

Contrast-enhanced CMRI can play a role in the differentiation between primary dilated cardiomyopathy and ischemic cardiomyopathy through the identification of myocardial scarring or fibrosis as presence of delayed enhancement. This would reduce the costs and inherent risk associated with invasive cardiac catheterization on which the diagnosis of cardiomyopathy is still dependent [3]. In addition, CMRI in non-ischemic cardiomyopathy patients strongly predicts adverse cardiac outcomes [4]. Cardiac magnetic resonance imaging is frequently used as a part of the cardiac workup and can be used to accurately identify the distribution of ejhm.journals.ekb.eg 3941 scar patterns, scar characterization, and measure the ventricular chamber size and function. The identification of MRI characteristics able to differentiate cardiac sarcoidosis from Arrhythmogenic Right Ventricular Dysplasia (ARVC) could prompt additional clinical testing and eventually lead to a better and more accurate diagnosis approach [1]. Cardiac cine MRI along with delayed myocardial enhancement MRI and other MRI pulse sequences can provide information more than echocardiography for tissue characterization. [1].

\section{Aim of the Work}

This study aims to identify the role of cardiac magnetic resonance imaging to diagnose various types of cardiomyopathy.

\section{Patients and Methods}

This study is an observational cross sectional study. It included 50 patients with suspected different types of nonischemic cardiomyopathies referred from outpatient clinic of Cardiology at Al-Hussein University hospital from November 2018 to July 2019.

Inclusion criteria:

Patients with different types of non-ischemic cardiomyopathies diagnosed by clinical data and echocardiography including the following:

1. Dilated Cardiomypathy.

2. Hypertrophic Cardiomyopathy.

3. Restrictive Cardiomyopthy.

4. Specific types of non-ischemic cardiomyopathy.

Exclusion criteria:

1. Patients with cardiomyopathy due to coronary artery disease.

2. Patients with cardiac devices (ICD, pace maker and CRT).

3. Patients with metallic implants Ex. Mechanical Prosthetic valves.

4. Patients with gadolinium hypersensitivity.

5. Patients with renal impairment of GFR below 30 $\mathrm{ml} / \mathrm{min}$.

6. Patient with Severe claustrophobia.

Ethical approval and written informed consent: An approval of the study was obtained from Al- Azhar University academic and ethical committee. Every patient signed an informed consent for acceptance of the operation.

Patient preparation and Set up:

No special preparation is needed before the assessment. Drugs are not to be discontinued. Initial, a clinical history was taken. Patients were then screened for contraindication to MR imaging. All undergarments containing nylon or metal were taken out. The previous may cause artifacts because of static electricity and the latter can cause image degradation. All steps of the study were explained in details for each patient including breath hold instructions. To evaluate patient's ability of breath holding for relatively long time; they were required to perform a deep inspiration and to continue to hold their breath without pushing (i.e., Valsalva maneuver). Sedation may be required in pediatric case. Some the patients had regular sinus rhythm while others were complaining of arrhythmia such as cases with ARVD, some cases of HCM and some of ischemic cases.

Magnetic Resonance Imager

The study was done at Radiology department of AlHussien university hospital, Al-Azhar University, Cairo, Egypt; during the study from January 2019 till July 2020, using a Philips Achieva (1.5 Tesla) superconducting magnet (Netherland). Patient Position All patients were studied in the supine position, head first. The patient's knees and legs could be elevated to help relieve back strain and secure the patient's comfort. The patients were offered cotton blankets for warmth. Head phones with the MRI machine were used to reduce repetitive gradient noise and in the same time allow the patients to hear the breath-hold instructions.

ECG Lead positioning: Four carbon fibers ECG pads were placed on the anterior chest wall, the first is placed $1 \mathrm{~cm}$ to the left of the xyphi-sternum, the second and the third are places in such a way that they are aligned at $90^{\circ}$ to each other where the first electrode forms the right angle and the distance between the electrodes $15 \mathrm{~cm}$. The fourth electrode is placed below the first electrode.

The ECG leads were attached. The green lead to the first pad, the red lead to the second pad, the white lead to the third pad, and the black lead to the fourth pad. The QRS complex is then checked on the MRI monitor, adjustments of the site of the leads is done accordingly. The patient's heart rate is also detected on MRI monitor; it is used to determine the cardiac frequency as it should be close to the patient's.

The Respiratory Sensor

The respiratory sensor was set over the most extreme zone of respiratory development (abdomen and chest) under the coil. A tie was utilized to fix the sensor. The respiratory signal was then checked as the respiratory wave showed up on the screen and was utilized to recognize the patient's respiratory beat and synchronize breath hold directions to the patient's capacities.

Respiratory sensor. The SENSE (affectability encoding) cardiac coil 6 element phased-array coil, receive only) was utilized. It has an inflexible lower part and flexible upper part. The lower part contains two phased array coil elements and the 
upper part contains four phased array elements. The coil is positioned on the chest. It is carefully strapped into the patient by four ties. The association with the magnet is checked.

Picture Acquisition Scout in the three planes without breath holding.

Planning vertical long axis image from the axial orthogonal image at the level of the left ventricle. Planning the horizontal long axis view from the vertical long axis view. Planning the short axis view from the horizontal long axis view Figure 1.

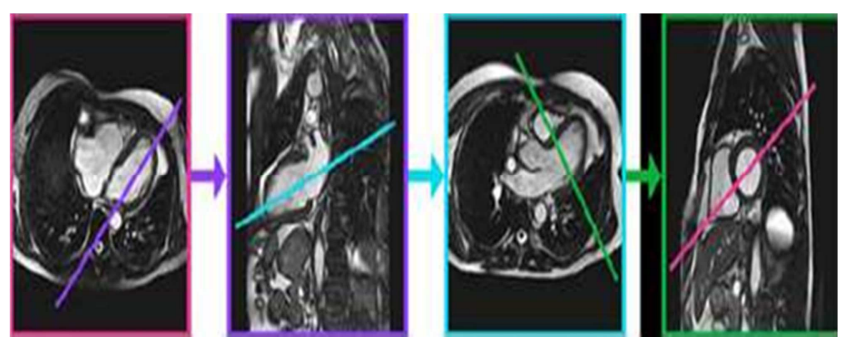

Figure 1. Planning of the cardiac views.

Data Analysis

Analysis of the CMR (DICOM) images was performed using Brilliance $170 \mathrm{P}$ workstation.

Left ventricular ejection fraction and volumes were measured automatically from the cine images after tracing of the endocardial border of the left ventricle in the short axis images at end systole and end diastole for each section.

Right ventricular volumes and ejection fraction were quantified by the same method for cases of pathologies related to right ventricle.

For regional wall motion analysis: cine MRI was evaluated. Wall motion abnormality was classified into:

Mild-to-moderate hypokinesia.

Severe hypokinesia.

Akinesia or dyskinesia.

Determination of the presence or absence of late gadolinium enhancement was done by reviewing all contrastenhanced images. Enhancement patterns were classified into subendocardial, transmural (more than $50 \%$ of wall thickness), subepicardial, midwall or pathymesocardial.

Statistical analysis:

acquired data were analyzed using the statistical package for social sciences, version 20.0 (SPSS Inc., Chicago, Illinois, USA). Quantitative data were expressed as mean \pm standard deviation (SD). Qualitative data were expressed as frequency and percentage.

The following tests were done:

Independent-samples t-test of significance was used when comparing between two means.

Chi-square (x2) test of significance was used in order to compare proportions between two qualitative parameters.

The confidence interval was set to $95 \%$ and the margin of error accepted was set to $5 \%$. The p- value was considered significant as the following:

Probability (P-value)

$\mathrm{P}$-value $<0.05$ was considered significant.

$\mathrm{P}$-value $<0.001$ was considered as highly significant.

$\mathrm{P}$-value $>0.05$ was considered insignificant.

\section{Results}

Our study included 50 patients with suspected different types of non-ischemic cardiomyopathy on basis of echocardiography, ECG changes or CT angiography of the coronary arteries. Some of them were referred for preoperative evaluation. Minor of them were referred for evaluation of ventricular masses accidentally discover during echocardiography.

Table 1. Cause of reference of cases.

\begin{tabular}{ll}
\hline Cause of refer & No. of patients \\
\hline Suspected CMP & 43 \\
Pre-operative evaluation & 4 \\
Vent. Masses & 3 \\
\hline
\end{tabular}

All patients were subjected to delayed enhancement cardiac MRI

Involves 50 patients that was suspected to have different causes of non-ischemic cardiomyopathies.

They were subdivided into.

Group A (primary cardiomyopathy: 32 patients; 64\%)

Group B (secondary cardiomyopathy: 18 patients; 36\%)

Different patterns of enhancement according to the following table.

Table 2. Showing different patterns of enhancement.

\begin{tabular}{lllll}
\hline & DE pattern & No. of cases & Percentage of cases \\
\hline Pattern 0 & No enhancement & 15 & 30 \\
Pattern I & patchymesocardial & 17 & 34 \\
Pattern II & uniform (midwall) mesocardial & 12 & 24 \\
Pattern III & subepicardial & 4 & 8 \\
Pattern VI & Striated mesocardial pattern (Zebra pattern) & 2 & 4 \\
\hline
\end{tabular}

Group A include 32 patients with primary cardiomyopathy not related to other causes and were distribute as follow.

Hypertrophic cardiomyopathy: 14 cases, $43,8 \%$ of group A cases.

Primary dilated cardiomyopathy: 7 cases, $21.9 \%$ of group A cases.
Primary restrictive cardiomyopathy: 3 case, $9 \%$ of group A cases.

Arrhythmogenic right ventricular dysplasia: 2 cases, $6.2 \%$ of group A cases.

Left ventricular non-compaction: 4 cases, $12.5 \%$ of group A cases. 
Tako-t subocardiomyopathy: 2 case, $6.3 \%$ of group A cases.

The study involves 14 cases of hypertrophic cardiomyopathy with excellent assessment of the morphological type of hypertrophic cardiomyopathy as well as its functional impact whether occurrence of left ventricular outflow obstruction, systolic anterior wall motion of the mitral valve as well as right ventricular involvement. Delayed enhancement reveals the extent of myocardial fibrosis and scarring.

The patients' age ranged from 1 year to 35 years old with main age 18.3 years. Their history varies from shortness of breath, poor feeding and weight gain in one patient (1 year old).

Other symptoms include recurrent chest pain with giant Qwave in ECG, repeated syncopal attacks, palpitation and easy fatigability.

Positive family history of unexplained sudden cardiac death was detected in 6 cases ( $40 \%$ of cases).

According to the morphological types:

Symmetrical type was seen in 4 cases of 14 cases $(28.6 \%)$.

Mid-ventricular type was seen in 3 cases of 14 cases (21.4\%).

Sigmoid type with reversed septal contour was seen in 3 cases of 14 cases $(21.4 \%)$.

Localized mass like form in 2 cases of 14 cases (14.2\%).

$\mathrm{Bi}$-ventricular involvement was seen in 2 cases of 14 cases (14.2\%).

The ejection fraction of these cases ranges from 38 to 77 (main 57.5).

The study contains 7 cases of primary dilated cardiomyopathy

They all presents with variable degree of left ventricular +/- right ventricular failure.

All showed the characteristic pattern AII of delayed enhancement.

All presented with impaired systolic function with ejection fraction 27.5 to 44.2 ; main ejection fraction (34.4).

All cases showed mild to moderate mitral regurge $(100 \%$ of cases), tricuspid regurge was seen in 3 cases and aortic regurge was seen in 4 cases ( $71 \%$ of cases).

The most value of MRI study of these patients is to differentiate whether if heart failure is related to 1ry dilated cardiomyopathy or other causes such as ischemia as they have different management in each groups.

Two cases of Arrhythmogenic right ventricular dysplasia has been reported in the study presenting with palpation, dyspnea, recurrent syncopal attacks. ECG changes done by Holter methods were suggestive of the disease.

All shows right ventricular dilatation with impaired right ventricular function, apical ballooning and evidence of variable degree of fibrofatty infiltration.

Four cases of primary restrictive cardiomyopathy were seen showing characteristic bi- atrial dilatation and impaired left ventricular diastolic function.

Evidence of pulmonary venous congestion was noted. Pericardial effusion and ascites was seen in two of these cases.

The history and work-up was unremarkable for any other causes of secondary restrictive cardiomyopathy. No sign of pericardial disease (constrictive pericarditis) was seen in our cases.

Five cases of left ventricular non-compaction were seen in the study and were accidentally discovered during routine echocardiographic evaluation.

Two cases of Tako-tsubo cardiomyopathy were seen showing characteristic apical ballooning with impaired left ventricular function.

ECG changes and invasive catheter were unremarkable.

These cases underwent another follow-up study 3 months later showing improvement of both functional and morphological appearances.

Group B include 18 patients with secondary cardiomyopathy related to known causes and were distributed as follow.

Toxic cardiomyopathy (alcoholic): 4 case, $22.2 \%$ of group b2 cases.

Endomyocardial fibrosis: 1 case, $5 \%$ of group b2 cases.

Iron overload related cardiomyopathy: 3 cases, $16.6 \%$ of group $\mathrm{b} 2$ cases.

Cardiac Amyloidosis: 3 cases, $16.6 \%$ of group b2 cases.

Cardiac sarcoidosis: 3 cases, $16.6 \%$ of group b2 cases.

Post-inflammatory cardiomyopathy: 4 cases, $22.4 \%$ of group b2 cases.

Different causes of dilated cardiomyopathy were demonstrated in table 3 .

Table 3. Showing causes of secondary cardiomyopathy.

\begin{tabular}{|c|c|c|c|}
\hline Cardiomyopathy & cause & No. of cases & $\%$ \\
\hline Toxic (alcoholic) cardiomyopathy & chronic alcohol consumption & 4 & 22.2 \\
\hline Endomyocardial fibrosis & Hyperesinophilic syndrome & 1 & 5 \\
\hline Iron overload & repeated blood transfusion & 3 & 16.6 \\
\hline Cardiac Amyloidosis & secondary amyloidosis in patient with chronic renal disease & 3 & 16.6 \\
\hline Cardiac sarcoidosis & secondary to sarcoid deposition with long-standing pulmonary sarcoidosis & 3 & 16.6 \\
\hline Post-inflammatory cardiomyopathy & Post-viral myocarditis and acute peri-myocarditis & 4 & 22.2 \\
\hline
\end{tabular}

The morphologic patterns of cardiomyopathy were:

Dilated pattern in 8 cases $44.4 \%$.

Hypertrophic pattern in 4 case $22.2 \%$.

Restrictive pattern in 3 case $16.6 \%$.

Mixed pattern in 3 case $16.6 \%$.
Associated findings were as follow:

Mitral regurge and tricuspid regurge detected in cases of dilated pattern.

Apical mural thrombus: characteristic of endomyocardial fibrosis. 
Pulmonary lesions, mediastinal lymphadenopathy and pericardial effusion in case of cardiac sarcoid.

Assessment of hepatic iron overload in the same study in case of chronic anemias.

Illustrative Cases

Case 1

Female Patients, 29y referred from cardiac outpatient clinic.

Echocardiography: left ventricular asymmetrical hypertrophic myocardium, $\mathrm{EF}=79 \%$.

CMR findings

Left ventricular asymmetrical myocardial hypertrophy, maximum wall thickness is noted at basal anterior wall of basal cavitary region that measures about $25 \mathrm{~mm}$.

Evidence of LVOT narrowing and SAM of mitral valve during rest with estimated gradient below $16 \mathrm{mmHg}$.

LGE left ventricular septal and inferior wall mesocardial patchy enhancement is noted involving the apical, midcavitary and basal levels of myocardium.

Mild mitral valve regurgitation is noted $(\mathrm{RF}=18 \%)$.

$\mathrm{LVM}=151 \mathrm{gm}$, Indexed LV values to BSA of $0.90=92$ $\mathrm{g} / \mathrm{m}^{2}$.

A 29-year-old female with HOCM and systolic anterior motion of the mitral valve causing subvalvular aortic stenosis and mitral regurgitation.

Case 2

A 43-year-old male with idiopathic dilated cardiomyopathy (DCM).

The four-chamber SSFP images acquired at $1.5 \mathrm{~T}$ shows a markedly dilated left ventricle (LV) and severely decreased LV systolic function. The calculated LV ejection fraction was
$21 \%$, DCE images show mid-myocardial enhancement in the interventricular septum

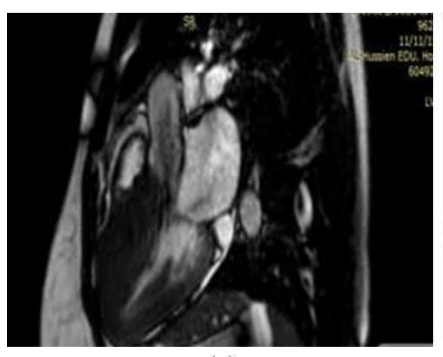

(a)

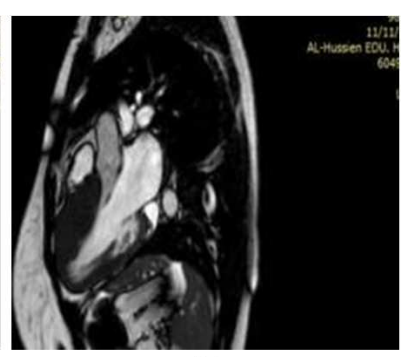

(b)
Figure 2. A three-chamber SSFP image acquired at 1.5T shows narrowing of the subvalvular left ventricular outflow tract (LVOT) during systole between the hypertrophied interventricular septum and the anterior leaflet of the mitral valve.

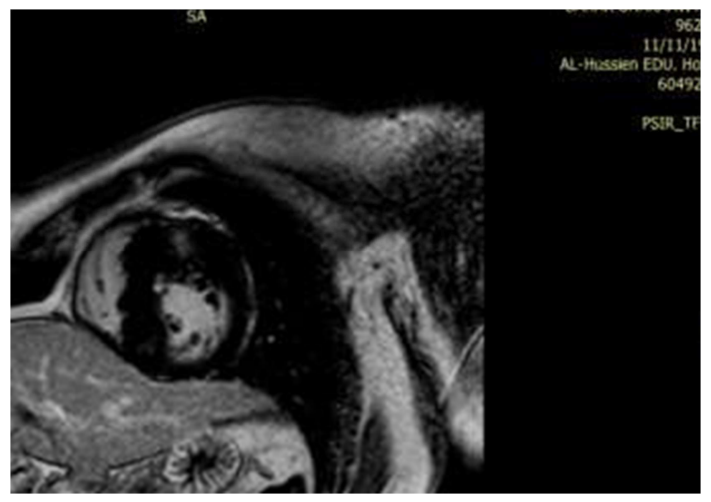

Figure 3. LGE: Short axis PSIR images showing patchy, mid myocardial pattern of enhancement in the interventricular septum, particularly near the insertion of the right ventricular free wall.

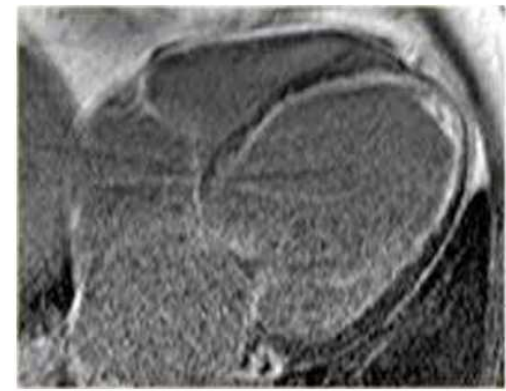

(a)

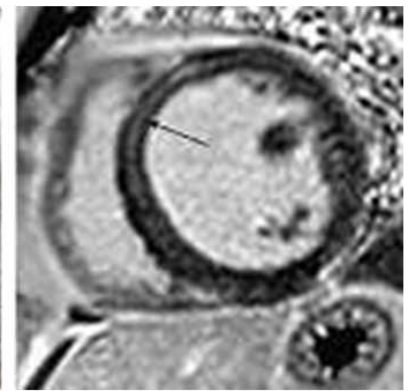

(b)

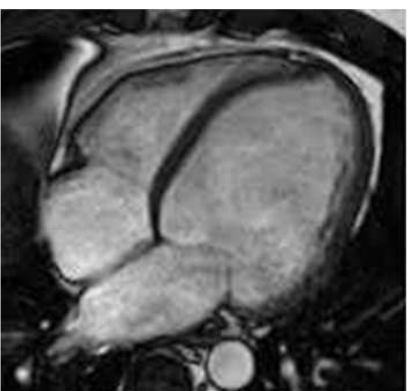

(c)

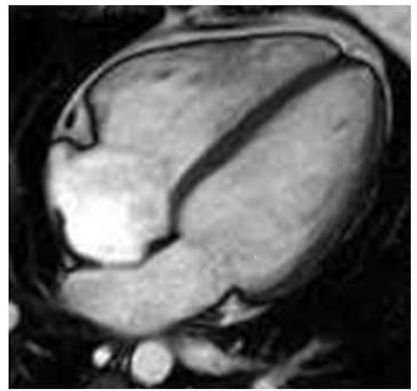

(d)

Figure 4. a: A four-chamber DCE image. b: Short-axis DCE image. c: A four-chamber SSFP image at end-diastole. D: A four-chamber SSFP image at endsystole show mid-myocardial enhancement in the interventricular septum.

\section{Discussion}

The current study demonstrates the feasibility of a time saving non-invasive MRI protocols that combines cine function, gadolinium-enhanced resting first-pass perfusion and Delayed enhancement CMR for diagnosis of nonischemic cardiomyopathies in a routine clinical setting. Our study was performed for 50 patients suspecting or known to have non ischemic cardiomyopathies. All patients underwent the CMR examination with no known complications.

The most recent study done by Coleman et al. [5], conclude that CMR appears to be highly relevant in the clinical as well as research evaluation of patients with overt as well as pre-clinical HCM. Late gadolinium enhancement allows tissue characterization of myocardial fibrosis. The method may potentially identify HCM patients at greatest risk for adverse cardiac events. CMR evaluation of HCM mutation carriers in an early stage of disease has yet to be extensively evaluated, but represents a promising method for exploring the inter- relationship between functional, morphologic and tissue abnormalities in HCM.

In our study, the morphological types were:

Symmetrical type was seen in 4 cases of 14 cases $(28.6 \%)$. 
Mid-ventricular type was seen in 3 cases of 14 cases (21.4\%).

Sigmoid type with reversed septal contour was seen in 3 cases of 14 cases $(21.4 \%)$.

Localized mass like form in 2 cases of 14 cases (14.2\%).

Bi-ventricular involvement was seen in 2 cases of 14 cases $(14.2 \%)$.

There was partial left ventricular outflow obstruction was seen in 6 cases $(42.9 \%)$. Systolic anterior wall motion (SAM) was seen in 7 cases $(50 \%)$.

And mitral stenosis was an association in one case (7.1\%).

The pattern of late enhancement varies from case to case with pattern $\mathrm{B} 0$ seen in three cases and pattern $\mathrm{BI}$ detected in eleven cases.

So, our study shows excellent assessment of the morphological type of hypertrophic cardiomyopathy as well as its functional impact whether occurrence of left ventricular outflow obstruction, systolic anterior wall motion of the mitral valve as well as right ventricular involvement. Delayed enhancement reveals the extent of myocardial fibrosis and scarring. The prognostic significance of the presence of LGE in HCM to adverse outcome is high. The presence of LGE in HCM patients has been associated with sudden cardiac death, systolic dysfunction and non-sustained ventricular tachycardia [6]. Multiple factors have been proposed in the etiology of myocardial fibrosis in HCM patients, although the true origin has not yet been determined. Ischemia may result from microvascular disease; increased end diastolic pressure together with the increased demand of LVH might initiate the processes of myocyte death and replacement fibrosis as a repair process [7].

Regarding dilated Cardiomyopathy (HCM):

Recognition of the underlying pathological substrate leading to ventricular dilatation may be crucial not only to specifically the target patients therapy (e.g. treatment of heart failure symptoms versus revascularization versus immunosuppressive and/or antiviral) but also for better individual risk stratification because of the extremely variable prognostic implications associated with the different forms of disease [8].

The study contains 7 cases of primary dilated cardiomyopathy presenting with variable degree of left ventricular \pm right ventricular failure and showed the characteristic pattern BII of delayed enhancement, the ejection fraction ranges from 27.5 to 44.2 ; main ejection fraction (34.4). All showed mild to moderate mitral regurge ( $100 \%$ of cases), tricuspid regurge was seen in 3 cases $(42 \%$ of cases) and aortic regurge was seen in two cases $(28 \%$ of cases).

The main clinical indication and value of MRI study of these patients is to differentiate whether if heart failure is related to 1ry dilated cardiomyopathy or other causes such as ischemia as they have different management in each groups.

Moreover, our study was excellent in differentiating primary from the secondary types of dilated cardiomyopathy, 7 cases that present with dilated pattern and revealed to be toxic (alcoholic) in 2 cases, Iron overload related cardiomyopathy in 2 case, Post-inflammatory cardiomyopathy in 3 cases.

Regarding restrictive Cardiomyopathy:

Our study included 3 cases of primary restrictive cardiomyopathy and one case of secondary cardiomyopathy due to endomyocardial fibrosis.

In these cases cardiac MRI shows excellent assessment in the first case differentiating it from constrictive pericarditis and visualization of the apical thrombi in the last case.

A study done by Jacquier et al. [9] stated that Cardiac MRI is a fundamental diagnostic tool because it helps in the differentiation between restrictive CMP and constrictive pericarditis, which have different therapeutic management since constrictive pericarditis requires surgical resection or stripping of the pericardium, whereas non-surgical management is used for RCM. Although reduced ventricular filling and diastolic volumes may be features of both diseases, pericardial thickening $(>4 \mathrm{~mm})$ is typical of constrictive pericarditis [9].

CMR showed that in restrictive CMP, septal convexity is maintained in all respiratory phases, where as in constrictive pericarditis, septal flattening can be observed in early inspiration [9].

Regarding Arrhythmogenic Right Ventricular Cardiomyopathy /Dysplasia:

Our study included two cases of ARVD with excellent assessment of right ventricular dilatation with impaired right ventricular function, apical ballooning and evidence of variable degree of fibrofatty infiltration.

ARVD remains an enigmatic disease and the diagnosis of ARVD is often challenging, especially at its early stages. Minor and major diagnostic criteria for ARVD have been proposed by the Task Force of the Working Group on Cardiomyopathies at 1994. These criteria encompass structural, histological, electrocardiographic, arrhythmic and genetic factors. On the basis of this classification, diagnosis of ARVD is fulfilled in the presence of two major criteria, one major plus two minor criteria, or four minor criteria from different groups [10-12].

Regarding Left Ventricular Non-Compaction:

The incidence in adults is about $0.05 \%$, in our study 5 cases were included. Birnie et al.[11], used diastolic steadystate free precession cine frames to determine the ratio of thickness of the trabecular and compact layers, the trabeculations being more easily identified by CMR in the relaxed heart. The diastolic ratio of 2.3 showed high diagnostic accuracy for distinguishing pathological LVNC from the degrees of non-compaction observed in healthy, dilated and hypertrophied hearts. $\mathrm{A} \mathrm{NC} / \mathrm{C}$ ratio of 2.3 in diastole distinguished pathological non-compaction, with values for sensitivity, specificity, positive, and negative predictions of $86 \%, 99 \%, 75 \%$, and $99 \%$, respectively [11].

Jacquier et al.[9] conclude that the value of trabeculated LV mass above $20 \%$ of the global mass of the LV predicted the diagnosis of LVNC. The method described is reproducible and provides an assessment of the global amount of LV trabeculation. A trabeculated LV mass above 
$20 \%$ of the global LV mass is highly sensitive and specific for the diagnosis of LVNC [9].

Regarding Amyloidosis:

Our study included one patient with cardiac amyloidosis in a patient with chronic renal failure that shows hypertrophic pattern. CMR was excellent in demonstrating the typical pattern of enhancement differentiating it from other causes of hypertrophic pattern $[12,13]$.

Detection of early stages, which may respond to therapy and exclusion of other disease mimicking amyloidosis appears crucial for patient's management $[14,15]$.

\section{Conclusion and Recommendation}

CMR is a valuable (minimally or non-invasive) imaging technique in patients with suspected or known to have non ischemic cardiomyopathies. It help to identify the underlying cause, predict prognosis and responsiveness to therapy and affect management as well follow-up and most recently as primary prevention diagnostic tool. Further researches should focus on each type of non-ischemic cardiomyopathy thus providing more diagnostic value with more benefit to the clinicians for management of their patients.

\section{References}

[1] O'Neill AC, McDermott S, Ridge CA et al. (2012): Investigation of cardiomyopathy using cardiac magnetic resonance imaging part 2: Rare phenotypes. World J Cardiol., 4 (5): 173-82.

[2] Bogaert J and Dymarkowski S (2012): Ischemic Heart. In: Clinical Cardiac MRI, Bogaert, J., S. Dymarkowski, A. M. Taylor and V. Muthurangu (Eds.), Springer, New York, Pp. 227-231.

[3] Hinojar R, Foote L, Sangle S et al. (2016): Native T1 and T2 mapping by CMR in lupus myocarditis: Disease recognition and response to treatment. International Journal of Cardiology, 222: 717-26.

[4] Chan RH, Maron BJ, Olivotto I et al. (2014): Prognostic value of quantitative contrast-enhanced cardiovascular magnetic resonance for the evaluation of sudden death risk in patients with hypertrophic cardiomyopathy. Circulation, 130: 484-95.

[5] Coleman GC, Shaw PW, Balfour PC et al. (2017): Prognostic Value of Myocardial Scarring on CMR in Patients With
Cardiac Sarcoidosis. JACC Cardiovascular Imaging, 10: 411420.

[6] White JA, Kim HW, Shah D et al. (2014): CMR imaging with rapid visual $\mathrm{T} 1$ assessment predicts mortality in patients suspected of cardiac amyloidosis. JACC Cardiovascular Imaging, 7: 143-56.

[7] McLellan A, Ellims AH, Prabhu S et al. (2016): Diffuse ventricular fibrosis on cardiac magnetic resonance imaging associates with ventricular tachycardia in patients with hypertrophic cardiomyopathy. Journal of Cardiovascular Electrophysiology, 27: 571-80.

[8] Gulati A, Jabbour A, Ismail TF et al. (2013): Association of fibrosis with mortality and sudden cardiac death in patients with nonischemic dilated cardiomyopathy. Jama., 309: 896908 .

[9] Jacquier A, Thuny F, Jop B et al. (2010): Measurement of trabeculated left ventricular mass using cardiac magnetic resonance imaging in the diagnosis of left ventricular noncompaction. European Heart Journal, 31: 1098-104.

[10] McKenna WJ, Desai MY, Ommen SR et al. (1994): Imaging phenotype versus genotype in hypertrophic cardiomyopathy. Circ Cardiovasc Imaging, 4: 156-68.

[11] Birnie DH, Sauer WH, Bogun Fet al. (2014): HRS expert consensus statement on the diagnosis and management of arrhythmias associated with cardiac sarcoidosis. Heart Rhythm, 11: 1305-23.

[12] Kramer CM, Appelbaum E, Desai MY et al. (2015): Hypertrophic Cardiomyopathy Registry: The rationale and design of an international, observational study of hypertrophic cardiomyopathy. American Heart Journal, 170: 223-30.

[13] Schieda N, Blaichman JI, Costa AF et al. (2018): GadoliniumBased Contrast Agents in Kidney Disease: A Comprehensive Review and Clinical Practice Guideline Issued by the Canadian Association of Radiologists. Can J Kidney Health Dis., $2054358118778573 . \quad$ doi: $10.1177 / 2054358118778573$.

[14] Te Riele AS, Tandri H, Bluemke DA (2014). Arrhythmogenic right ventricular cardiomyopathy (ARVC): cardiovascular magnetic resonance update. Journal of cardiovascular magnetic resonance: official journal of the Society for cardiovascular Magnetic Resonance; 16: 50.

[15] Vogelsberge H, Mahrholdt H, Deluigi CC, et al (2008). Cardivascular magnetic resonance in clinically suspected cordial amyloidosis: noninvasive imaging compared to endomyocardial biopsy. Journal of the American collage of Cardiology: 1022-30. 